\title{
Intramucosal gastric adenocarcinoma of poorly differentiated type in the young is characterized by Helicobacter pylori infection and antral lymphoid hyperplasia
}

\author{
Minako Hirahashi ${ }^{1,2}$, Takashi Yao ${ }^{1}$, Takayuki Matsumoto ${ }^{2}$, Ken-ichi Nishiyama ${ }^{3}$, \\ Masafumi Oya ${ }^{4}$, Mitsuo Iida ${ }^{2}$ and Masazumi Tsuneyoshi ${ }^{1}$ \\ ${ }^{1}$ Department of Anatomic Pathology, Pathological Sciences, Graduate School of Medical Sciences, Kyushu \\ University, Fukuoka, Japan; ${ }^{2}$ Department of Medicine and Clinical Science, Graduate School of Medical \\ Sciences, Kyushu University, Fukuoka, Japan; ${ }^{3}$ Pathology Division of National Hospital Organization Kyushu \\ National Cancer Center, Fukuoka, Japan and ${ }^{4}$ Pathology Division of Aso-Iizuka Hospital, Fukuoka, Japan
}

\begin{abstract}
The aim of this investigation was to clarify the histological characteristics of gastric cancer in the young. Twenty-three surgically resected specimens of young patients (under 30 years of age; young group) with intramucosal cancer of poorly differentiated type and $\mathbf{4 2}$ surgically resected specimens of elderly patients (more than $\mathbf{4 0}$ years of age; elderly group) with tumors of the identical depth and histological type were examined. The degree of gastritis and Helicobacter pylori (H. pylori) infection was evaluated according to the updated Sydney system. The incidence of $\boldsymbol{H}$. pylori infection was significantly higher in the young group than in the elderly group (96 vs $36 \%, P<0.05$ ). Within the background mucosa, antral chronic inflammatory infiltrates with lymphoid-follicle hyperplasia were more severe, and intestinal metaplasia was less frequent in the young group than in the elderly group. Glandular atrophy was not different between the two groups. Intramucosal gastric adenocarcinomas of poorly differentiated type in the young may be associated with $\mathrm{H}$. pylori infection with antral chronic inflammation with lymphoid-follicle hyperplasia, regardless of the existence of intestinal metaplasia within the background gastric mucosa.

Modern Pathology (2007) 20, 29-34. doi:10.1038/modpathol.3800714; published online 13 October 2006
\end{abstract}

Keywords: intramucosal gastric cancer; poorly differentiated adenocarcinoma; young patients; Helicobacter pylori infection; background mucosa

Gastric cancer is a common malignant neoplasm throughout the world, and it is one of the most common cancers within the Japanese population. ${ }^{1,2}$ In an analysis of 107 young patients aged 30 years or less, Nakamura et $a l^{1}$ from our department reported that the prevalence in female subjects was more frequent than in males, that poorly differentiated adenocarcinoma was more frequent than welldifferentiated adenocarcinoma, and that cancers occurred more frequently in the middle third of the stomach, when compared to cancers in the

Correspondence: $\mathrm{Dr} \mathrm{T}$ Yao, MD, Department of Anatomic Pathology, Graduate School of Medical Sciences, Kyushu University, Maidashi 3-1-1, Higashi-ku, Fukuoka 812-8582, Japan.

E-mail: takyao@surgpath.med.Kyushu-u.ac.jp

Received 2 May 2006; revised 1 September 2006; accepted 7 September 2006; published online 13 October 2006 elderly patients. These observations conform to those reported previously, ${ }^{1-3}$ and these characteristic clinicopathological features suggest that gastric cancer in young patients occurs under peculiar conditions.

Helicobacter (H.) pylori is considered to be a risk factor for the development of gastric cancer. ${ }^{4,5}$ Although $H$. pylori infection has been shown to be closely associated with the occurrence of welldifferentiated adenocarcinoma in the stomach of middle-aged or elderly patients, ${ }^{6}$ the contribution of the infection to the development of gastric cancer in young patients has not been elucidated, simply because the incidence of gastric cancer is low in the young. ${ }^{1,2,7}$ Even in pathological investigations, there have been only sparse data regarding the characteristics of the background gastric mucosa in young patients with intramucosal gastric cancer. In this study, we investigated the background mucosa of 
intramucosal gastric cancer in a young group and compared them with those in an elderly group with identical histology and invasion depth.

\section{Materials and methods}

During a period from July 1973 to December 2003, a total of 10771 cases of surgically resected gastric cancer were registered at the Department of Anatomic Pathology, Graduate School of Medical Sciences, Kyushu University. Among those patients, 109 patients ranged from 7 to 30 years of age at the time of surgery. Among these 109 cases, 23 (21\%) had solitary intramucosal gastric cancer. All these cases had gastric cancer of poorly differentiated type. These 23 cases were regarded as the young group. There were 2250 cases of intramucosal gastric cancer in patients aged 40 years or more, registered at our institute during the same three decades. Of those, 287 cases $(12.8 \%)$ had solitary intramucosal gastric cancer of poorly differentiated type. For the purpose of comparison with the young patients, we randomly chose 42 patients (41-76 years of age; the elderly group) from among these 287 patients.

The resected tissue specimens were fixed in 10\% buffered formalin and embedded in paraffin. Histological sections cut from the paraffin blocks were routinely stained with hematoxylin and eosin. We classified the macroscopic and histological features of gastric cancers, according to the Japanese classification of gastric cancer.

\section{Evaluation of Background Chronic Gastritis}

We observed the non-neoplastic mucosa within $5 \mathrm{~mm}$ from the margins of carcinoma cells as the gastric mucosa around the site of cancer. In addition, the background mucosa was defined as non-neoplastic mucosa of the lesser curvature of the corpus in cases where the cancer was located in the corpus, and as non-neoplastic mucosa from the lesser curvature of the antrum in cases where the cancer was located in the antrum. We then evaluated the following five items: (1) $H$. pylori density, (2) polymorphonuclear neutrophil activity, (3) degree of chronic inflammation, (4) degree of glandular atrophy and (5) degree of intestinal metaplasia, according to the updated Sydney system (1994). ${ }^{8}$ $H$. pylori infection was observed by modified Giemsa stain and immunohistochemical stain. In addition, we also evaluated lymphoid follicles. We counted numbers of lymphoid follicles and/or marked lymphoid aggregates in the gastric mucosa. Lymphoid-follicle hyperplasia was determined when we found more than two large lymphoid follicles with germinal center in the mucosa. All items were graded as follows: 0 , normal; 1 , mild; 2 , moderate and 3 , severe or marked.

\section{Statistical Analyses}

All the data from both groups were analyzed by the Fisher's exact test. A $P$-value of less than 0.05 was considered to be statistically significant.

\section{Results}

The clinicopathological features of the young and the elderly groups are summarized in Table 1 . The mean age of the young group was 27.3 years old and the group consisted of five male and 18 female subjects. The mean age of the elderly group was 56.3 years old and the group consisted of 24 male and 18 female subjects. Female subjects were more frequent in the young group than in the elderly group $(P<0.001)$. In the young group, one lesion was located in the upper third of the stomach, 16 in the middle third and six in the lower third. In the elderly patients, 34 lesions were in the middle third of the stomach and eight in the lower third of the stomach.

The mean diameter of tumors was $3.7 \mathrm{~cm}$ in the young group and it was $3.6 \mathrm{~cm}$ in the elderly group. In each group, macroscopic types of the cancers were all depressed type. The histological type of the cancer was poorly differentiated adenocarcinoma in 18 patients and signet-ring cell carcinoma in five patients in the young group. In elderly group, there were 34 cases of poorly differentiated adenocarcinoma and eight cases of signet-ring cell carcinoma.

Table 1 Clinicopathological features of young and elderly patients with intramucosal gastric cancer

\begin{tabular}{lcc}
\hline & Young group (n=23) & Elderly group (n=42) \\
\hline Age (years) & $21-30$ (mean 27) & $41-76($ mean 56$)$ \\
Gender (M:F) & $5: 18^{*}$ & $24: 18^{*}$ \\
Site of tumor (U:M:L) & $1: 16: 6$ & $0: 34: 8$ \\
Size of tumor (cm) & $0.5-6$ (mean 3.7) & $23: 0$ \\
Macroscopic type (depressed:elevated) & $18: 5$ & $0.7-8.6(\mathrm{mean} 3.6)$ \\
Histological type (poorly:signet-ring cell) & $2: 21$ & $42: 0: 0$ \\
Lymphatic permeation (+:-) & $0: 23$ & $34: 8: 0$ \\
Venus invasion (+:-) & $1: 41$ \\
\end{tabular}

${ }^{*} P<0.001$ 
Two cases in the young group and a case in the elderly group showed lymphatic permeation. There was not any case of venous invasion in each group. Thus, there was no statistical difference in tumor location, tumor size, macroscopic type, lymphatic permeation and vascular invasion between the two groups.

Histology of adjacent mucosa is compared in Table 2. $H$. pylori was histologically observed in 22 of 23 patients (96\%) in the young group but it was positive in only 15 of 42 patients in the elderly group (36\%, P<0.001) (Table 2). As shown in Table 2, grades for neutrophilic activity, glandular atrophy and lymphoid follicles were not different between the groups. In contrast, grades for chronic inflammatory infiltrates were more advanced in the young group than in the elderly group $(P=0.02)$. The scores for intestinal metaplasia were lower in the young group than in the elderly group $(P<0.001)$.

As an extended gastric resection was performed in 11 cases of the young group and 22 case of the elderly group, we could assess both corporal and antral mucosa in these cases. The comparison of the background mucosa of the cases is indicated in Table 3. In both the corpus and in the antrum, the density of $H$. pylori was higher in young patients was significantly higher than in elderly patients $(P<0.0001$ at both sites $)$. In contrast, score for intestinal metaplasia was significantly lower in the young than in the elderly at both sites.

Table 2 Degree of gastritis within the gastric mucosa adjacent to the site of intramucosal cancer

\begin{tabular}{lccc}
\hline & $\begin{array}{c}\text { Young } \\
\text { group } \\
(\mathrm{n}=23)\end{array}$ & $\begin{array}{c}\text { Elderly } \\
\text { group } \\
(\mathrm{n}=42)\end{array}$ & P-value \\
\hline H. pylori infection (0/1-3) & $22 / 1$ & $15 / 27$ & $<0.001$ \\
Neutrophil activity (0-1/2-3) & $14 / 9$ & $30 / 12$ & $\mathrm{NS}$ \\
Chronic inflammation (0-1/2-3) & $4 / 19$ & $20 / 22$ & 0.02 \\
Atrophy (0-1/2-3) & $20 / 3$ & $30 / 12$ & $\mathrm{NS}$ \\
Intestinal metaplasia (0/1-3) & $8 / 15$ & $35 / 7$ & $<0.001$ \\
Lymphoid follicles (0-1/2-3) & $15 / 8$ & $27 / 15$ & $\mathrm{NS}$ \\
\hline
\end{tabular}

The degree of hyperplastic lymphoid follicles in the antral mucosa was significantly higher in the young group than in the elderly group $(P=0.0004)$. Several large-sized lymphoid follicles were seen within the antral background mucosa in 9 of 11 young patients $(82 \%)$. These lymphoid follicles in the lamina proplia showed prominent germinal center and was large in size (Figure 1).

Neither neutrophilic activity nor chronic inflammation was different at any site between the two groups. However, there was a trend towards severe chronic inflammatory infiltrates and less glandular atrophy and intestinal metaplasia in the young group (Figure 2a and b), and a trend towards mild chronic inflammatory infiltrates and severe glandular atrophy and intestinal metaplasia in the elderly group (Figure 3a and b). Score for atrophic glands for the corporal mucosa tends to be higher in the elderly group than in the young group $(P=0.06)$. The degree of neutrophilic activity and chronic inflammatory infiltrates was not significantly different between the two groups at any site of the stomach.

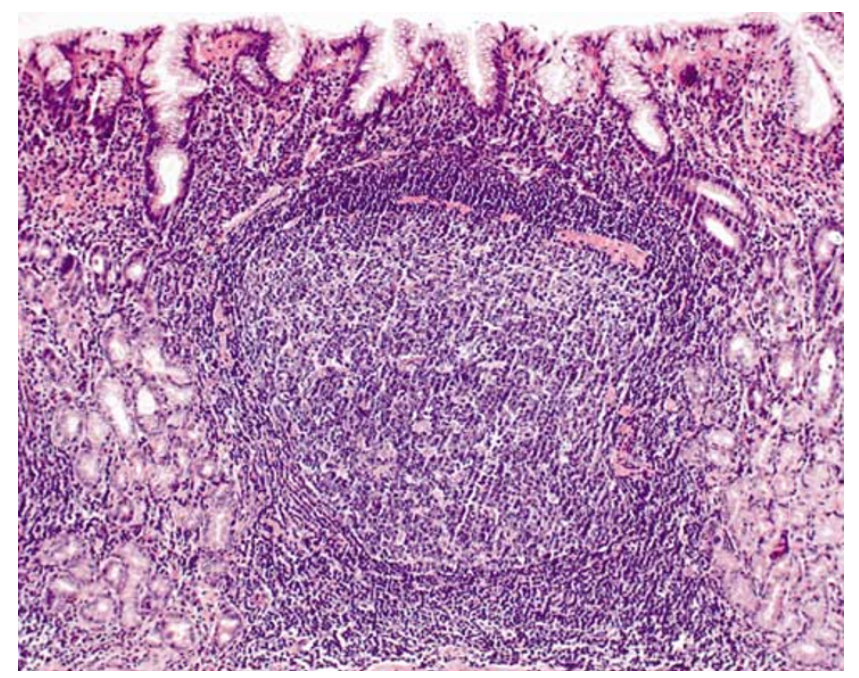

Figure 1 Lymphoid follicle hyperplasia in the antral mucosa of a 25-year-old young female subject. A big lymphoid follicle was seen in the lamina propria.

Table 3 Grading of gastritis within the background mucosa

\begin{tabular}{|c|c|c|c|c|}
\hline & \multicolumn{2}{|c|}{ Corpus } & \multicolumn{2}{|c|}{ Antrum } \\
\hline & Young group $(\mathrm{n}=11)$ & Elderly group $(\mathrm{n}=22)$ & Young group $(\mathrm{n}=11)$ & Elderly group $(\mathrm{n}=22)$ \\
\hline H. pylori infection & $2.09 \pm 0.94^{*}$ & $0.77 \pm 0.97^{*}$ & $1.18 \pm 0.98^{* *}$ & $0.36 \pm 0.73^{* *}$ \\
\hline Neutrophil activity & $1.45 \pm 0.82$ & $1.36 \pm 0.73$ & $1.36 \pm 0.67$ & $1.05 \pm 0.65$ \\
\hline Chronic inflammation & $2.00 \pm 0.78$ & $1.82 \pm 0.50$ & $2.00 \pm 0.63$ & $1.64 \pm 0.58$ \\
\hline Atrophy & $0.18 \pm 0.41$ & $0.73 \pm 0.88$ & $0.64 \pm 0.81$ & $1.00 \pm 0.82$ \\
\hline Intestinal metaplasia & $0.64 \pm 0.92^{\dagger}$ & $1.82 \pm 0.96^{\dagger}$ & $0.91 \pm 1.14^{\ddagger}$ & $1.86 \pm 1.08^{\ddagger}$ \\
\hline Lymphoid follicles & $1.46 \pm 0.69$ & $1.46 \pm 0.97$ & $2.36 \pm 0.81^{\S}$ & $1.23 \pm 0.75^{\S}$ \\
\hline
\end{tabular}

${ }^{*} P=0.0008 ;{ }^{*}{ }^{*} P=0.01 ;{ }^{\dagger} P=0.002 ;{ }^{\ddagger} P=0.02 ;{ }^{\circledR} P=0.0004$ 

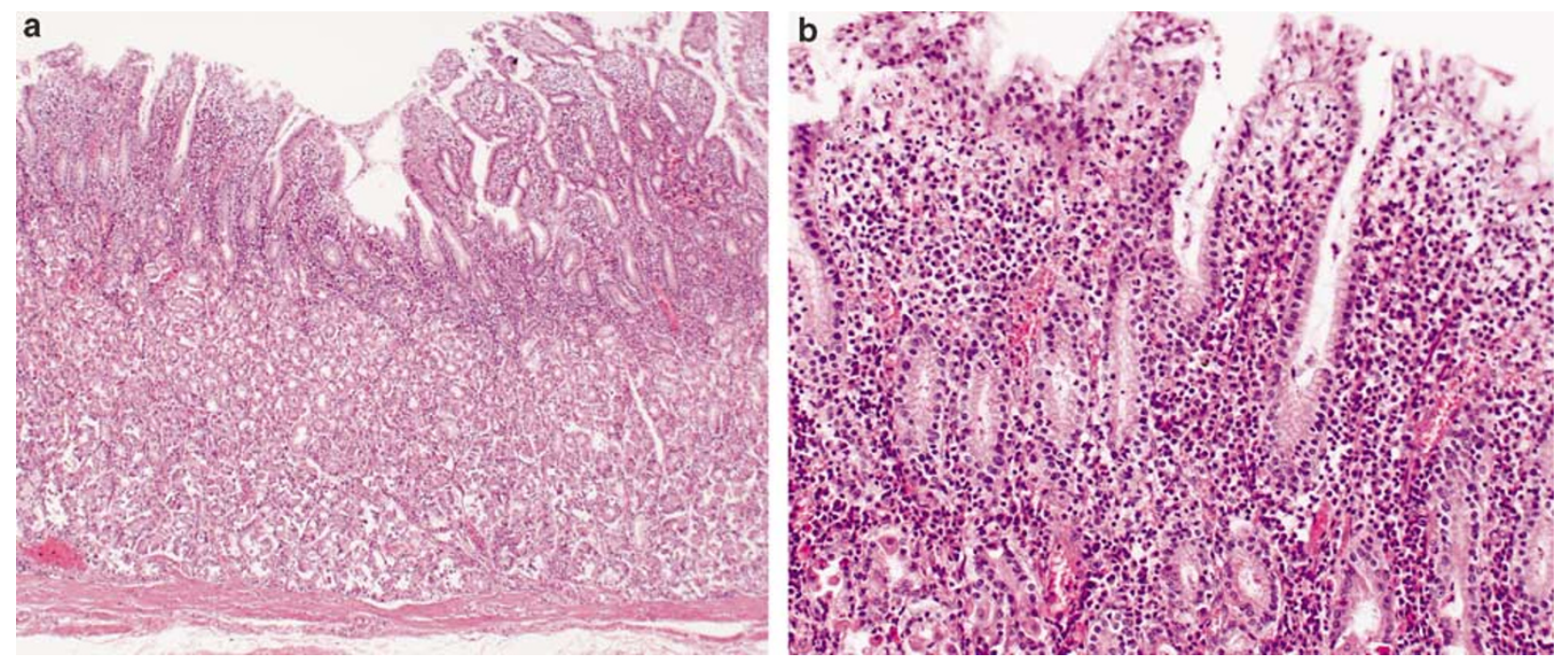

Figure 2 A common histological feature of the background mucosa in the young group. (a) Twenty-eight-year-old female subject. The corporal mucosa shows gastric proper foveolae and the layer of fundic glands at the 2/3 thickness of the mucosa (atrophy 0 , intestinal metaplasia 0). (b) Twenty-eight-year-old female subject (same case as a). The corporal mucosa shows severe chronic inflammatory infiltrate. Polymorphonuclear neutrophils are also moderately infiltrated in the mucosa (chronic inflammation $3+$, neutrophilic activity $2+$ ).
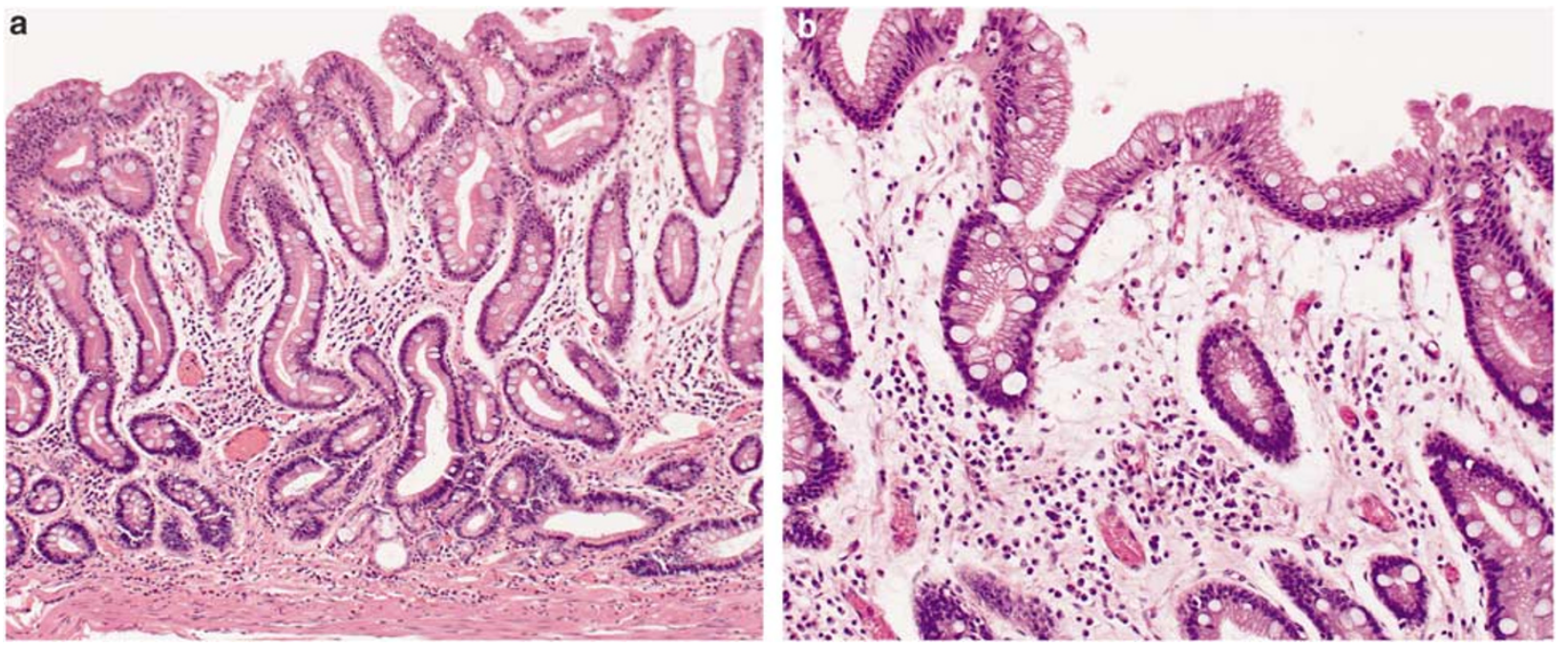

Figure 3 A common histological feature of the background mucosa in the elderly group. (a) Fifty-seven-year-old male subject. The corporal mucosa shows severe atrophy of fundic glands and foveolae with intestinal goblet cells (atrophy $3+$, intestinal metaplasia $3+$ ). (b) Fifty-seven-year-old male subject (same case as a). The corporal mucosa shows mild chronic inflammatory infiltrate. Polymorphonuclear neutrophils are not seen in the mucosa (chronic inflammation $1+$, neutrophilic activity 0 ).

\section{Discussion}

To evaluate carcinogenesis of gastric cancer, it is important to re-evaluate histological relationship between intramucosal gastric cancer and background gastric mucosa, in detail. There has been no report that analyzed only intramucosal gastric cancer and background gastric mucosa in the young. Our present investigation seems to be the first and an important report that elucidated the histopathological carcinogenesis of gastric cancer in the young.

In our exclusive cases of intramucosal cancers, the similar tendency was found as reported by
Nakamura et al, ${ }^{1}$ that female subjects were more frequent than male, poorly differentiated adenocarcinoma was more frequent than well differentiated one, and that cancers occurred more frequently in the middle third of the stomach, when compared to cancers in the elderly patients. In several studies, the clinicopathological features of gastric cancer in the young were somewhat different from ours. ${ }^{1,2,9-14,3,15-19}$ With regard to histological type, poorly differentiated adenocarcinoma tends to be more frequent in young adults, ${ }^{12-14,3}$ however, there is no difference in sex distribution between the young and elderly patients. ${ }^{9,10,13,14}$ The discrepancy 
in sex distribution between our and other reports was probably due to the ages of 'young' patients. Our patients under 30 years old were much younger than those of other reports, which dealt the age of under $40^{9-12,15,18}$ or $35^{13,14}$ years as the young group. Therefore, our present materials are considered to be more suitable for analysis of carcinogenesis of cancer in young patients.

The prevalence of $H$. pylori infection has already been reported to have relevance to gastric cancer in young patients. ${ }^{15-19}$ Haruma et $a l^{16}$ reported that the prevalence of $H$. pylori infection was higher in patients under 30 years of age with gastric cancer than in age- and sex-matched controls without gastric cancer. In addition, they also mentioned that the prevalence was not associated with tumor location, tumor stage or histological type. However, most of their materials revealed poorly differentiated adenocarcinoma and $95 \%$ of the poorly differentiated adenocarcinoma cases were positive for $H$. pylori infection. ${ }^{16}$ These observations together with the results of the current investigation strongly suggest that $H$. pylori infection contributes to the development of gastric cancer of poorly differentiated type in the young.

$H$. pylori infection gives rise to chronic inflammation, glandular atrophy and intestinal metaplasia in the gastric mucosa. ${ }^{20-22}$ Jaskiewicz et $a l^{23}$ reported that male subjects over 40 years of age are likely to demonstrate antral glandular atrophy, and patchy or diffuse intestinal metaplasia. With regard to glandular atrophy and intestinal metaplasia of the background mucosa, our young patients were mostly infected by $H$. pylori, however, they had less degree of corporal glandular atrophy and less frequent intestinal metaplasia around the intramucosal gastric cancer than elderly patients did. This result is consistent with that of the previous report by Mori et al. ${ }^{2}$

On the other hand, Koshida et $a l^{18}$ reported that there was significantly higher prevalence of $H$. pylori infection, glandular atrophy and intestinal metaplasia in cases with gastric cancer than in cases without gastric cancer in young patients. This result is not consistent with the above ones including ours, however, Koshida et $a l^{18}$ treated with patients younger than 40 years old (total of 40 patients, including only nine patients under 30 years of age). The differences between our results and those of Koshida et $a l^{18}$ is considered to be due to the difference in patient ages. Considering these findings, $H$. pylori infection is strongly related to outbreak of gastric cancer in young patients regardless of glandular atrophy and intestinal metaplasia.

There have been some reports demonstrating that pangastritis associated with a high lymphoid follicle density is seen in young patients with $H$. pylori infection. ${ }^{24-26}$ Genta et $a l^{25}$ and Miyamoto et $a l^{26}$ recently reported a possible association between gastric cancer and gastric lymphoid follicles ${ }^{25}$ or 'nodular gastritis' in the young. ${ }^{26}$ Nodular gastritis is a peculiar type of $H$. pylori-related gastritis characterized by gooseflesh appearance in the antral mucosa. Histopathologically, the nodules are composed of lymphoid follicles in the lamina propria. ${ }^{25,26}$ In our study, the antral mucosa of young patients showed a higher density of lymphoid follicles than that seen in elderly patients. This result may reflect that many of our cases also demonstrated 'nodular gastritis' associated with $H$. pylori infection.

In conclusion, our pathological analysis revealed that $H$. pylori infection was strongly related to the development of gastric cancer in young patients. This observation strongly supports an additional pathway of $H$. pylori-related gastric carcinogenesis, that is, independent of glandular atrophy or intestinal metaplasia. Thus, if young people are infected by $H$. pylori and reveal 'nodular gastritis', the eradication of $H$. pylori is recommended.

\section{Acknowledgements}

The English used in this manuscript was revised by Miss K Miller (Royal English Language Centre, Fukuoka, Japan).

\section{References}

1 Nakamura T, Yao T, Niho Y, et al. A Clinicopathological study in young patients with gastric carcinoma. J Surg Oncol 1999;71:214-219.

2 Mori M, Sugimachi K, Ohiwa T, et al. Early gastric carcinoma in Japanese patients under 30 years of age. Br J Surg 1985;72:289-291.

3 Kim DY, Ryu SY, Kim YJ, et al. Clinicopathological characteristics of gastric carcinoma in young patients. Langenbecks Arch Sur 2003;388:245-249.

4 Warren JR. Unidentified curved bacilli on gastric epithelium in active chronic gastritis. Lancet 1983;1: 1273-1275.

5 Uemura N, Okamoto S, Yamamoto S, et al. Helicobacter pylori infection and the development of gastric cancer. New Engl J Med 2001;345:784-789.

6 Loffeld RJLF, Willems I, Flendrig JA, et al. Helicobacter pylori and gastric carcinoma. Histopathology 1990;17: 537-541.

7 Fenoglio-Preiser C, Carneiro F. Gastric carcinoma. In: Hamilton SR, Aaltonen LA (eds). Tumor of the Stomach; Gastric Carcinoma. Pathology and Genetics of Tumours of the Digestive System. World Health Organization Classification of Tumours: Lyon, 2000, pp 39-52.

8 Dixon MF, Genta RM, Yardley JH, et al, the participants in the international workshop on the histopathology of gastritis, Houston 1994. Classification and grading of gastritis: the update Sydoney system. Am J Surg Pathol 1996;20:1161-1181.

9 Koea JB, Karpeh MS, Brennan MF. Gastric cancer in young patients: demographic, clinicopathological and prognostic factors in 92 patients. Ann Surg Oncol 2000;7:346-351. 
10 Medina-Franco H, Heslin MJ, Cortes-Gonzalez R. Clinicopathological characteristics of gastric carcinoma in young and elderly patients: a comparative study. Ann Surg Oncol 2000;7:515-519.

11 Rugge M, Busatto G, Cassaro M, et al. Patients younger than 40 years with gastric carcinoma. Helicobacter pylori genotype and associated gastritis phenotype. Cancer 1999;85:2506-2511.

12 Radi MJ, Fenoglio-Preiser CM, Bartow SA, et al. Gastric carcinoma in the young: A clinicopathological and immunohistochemical study. Am J Gastroenterol 1986;81:747-756.

13 Tso PL, Bringaze WL, Dauterive AH, et al. Gastric carcinoma in the young. Cancer 1987;59:1362-1365.

14 Holburt E, Freedman SI. Gastric carcinoma in patients younger than age 36 years. Cancer 1987;60:1395-1399.

15 Ramos-De la Medina A, Salgado-Nesme N, TorresVillalobos G, et al. Clinicopathologic characteristics of gastric cancer in a young patient population. J Gastrointest Surg 2004;8:240-244.

16 Haruma K, Komoto K, Kamada T, et al. Helicobacter pylori infection is a major risk factor for gastric carcinoma in young patients. Scand J Gastroenterol 2000;35:255-259.

17 Kokkola A, Valle J, Haapiainen R, et al. Helicobacter pylori infection in young patients with gastric carcinoma. Scand J Gastroenterol 1996;31:643-647.

18 Koshida Y, Koizumi W, Sasabe M, et al. Association of Helicobacter pylori-dependent gastritis with gastric carcinomas in young Japanese patients: histopathological comparison of diffuse and intestinal type cancer cases. Histopathology 2000;37:124-130.
19 Chang-Claude J, Raedsch $\mathrm{R}$, Waldherr $\mathrm{R}$, et al. Prevalence of Helicobacter pylori infection and gastritis among young adults in China. Euro J Cancer Preven 1995;4:73-79.

20 Baldini L, Pretolani S, Bonvicini F, et al. Effect of Helicobacter pylori infection, age and epithelial cell turnover in a general population at high risk for gastric cancer. Panminerva Med 1999;41:187-192.

21 Storskrubb T, Aro P, Ronkainen J, et al. A negative Helicobacter pylori serology test is more reliable for exclusion of premalignant gastric conditions than a negative test for current $H$. pylori infection: a report on histology and $\mathrm{H}$. pylori detection in the general adult population. Scand J Gastroenterol 2005;40:302-311.

22 Negrini R, Savio A, Poiesi C, et al. Antigenic mimicry between Helicobacter pylori and gastric mucosa in the pathogenesis of body atrophic gastritis. Gastroenterology 1996;111:655-665.

23 Jaskiewicz K, Louwrens HD. Chronic atrophic gastritis in a population at risk for gastric carcinoma. Anticancer Res 1991;11:835-840.

24 Sepulveda AR, Peterson LE, Shelton J, et al. Histological patterns of gastritis in $\mathrm{H}$. pylori-infected individuals with family history of gastric cancer. Am J Gastroenterol 2002;97:1365-1370.

25 Genta RM, Hamner HW, Graham DY. Gastric lymphoid follicles in Helicobacter pylori infection: frequency, distribution and response to triple therapy. Hum Pathol 1993;24:577-583.

26 Miyamoto M, Haruma K, Yoshihara M, et al. Nodular gastritis in adults is caused by Helicobacter pylori infection. Dig Dis Sci 2003;48:968-975. 